\title{
Osteoarticular manifestations as initial symptoms of WD with novel compound heterozygote mutations in the ATP7B gene: a case report
}

\author{
Jia $\mathrm{Li}^{1 \# \wedge}$, Qing Lu${ }^{2 \# \wedge}$, Jianyu $\mathrm{Yu}^{1}$, Min $\mathrm{Ji}^{3}$, Liangjing $\mathrm{Lu}^{1 \wedge}$ \\ ${ }^{1}$ Department of Rheumatology, Ren Ji Hospital, Shanghai Jiao Tong University School of Medicine, Shanghai, China; ${ }^{2}$ Department of Radiology, \\ Ren Ji Hospital, Shanghai Jiao Tong University School of Medicine, Shanghai, China; ${ }^{3}$ Department of Radiology, Children's Hospital of Fudan \\ University, Shanghai, China \\ \#These authors contributed equally to this work. \\ Correspondence to: Liangjing Lu, MD, PhD. Department of Rheumatology, Ren Ji Hospital, Shanghai Jiao Tong University School of Medicine. 145, \\ Middle Shandong Road, Shanghai 200001, China. Email: lu_liangjing@163.com.
}

\begin{abstract}
Wilson disease (WD) is a rare autosomal recessive disease characterized by hepatic, neurologic and psychiatric, and variable manifestations. Skeletal and articular manifestations are usually overlooked at an early stage in WD patients, which have an effect on therapeutic outcome and prognosis. We report a 13-yearold girl of Chinese Han ethnicity with arthralgia and fracture as initial symptoms of WD. Laboratory tests showed her 1:80 of antinuclear antibodies (ANA). The patient was diagnosed with oligoarticular juvenile idiopathic arthritis (JIA) and treated with $10 \mathrm{mg}$ of methotrexate (MTX) every week and diclofenac sodium every day. Her symptoms showed no improvement over 6 months and her medications were ceased. Then the patient presented to our department with a 3-week history dysarthria, gait abnormalities, dystonia and tremor. Kayser-Fleischer rings, serum ceruloplasmin and liver biopsy confirmed the diagnosis of WD. Genetic analysis was performed using SureSelect Clinical Research Exome v2 and then verified by bidirectional Sanger sequencing. We found that the patient carried a novel compound heterozygous mutation in ATPase copper transporting beta $(A T P 7 B)$ on both chromosomes, which consist of a heterozygote of NM_000053.3 (ATP7B): c.3884C>T p. Ala1295Val and large fragment heterozygous deletion in exons 10-11 of $A T P 7 B$ gene identified using multiplex ligation-dependent probe amplification (MLPA), to be inherited from her asymptomatic parents, respectively. The patient's symptoms were relieved at the 1-year followup after treatment with D-penicillamine and oral zinc. This case highlights that WD should be taken into consideration in adolescents with unexplained joint pain, arthritis, and fracture of the large joints.
\end{abstract}

Keywords: Osteoarticular manifestations; Wilson disease (WD); ATP7B; case report

Submitted Oct 27, 2020. Accepted for publication Feb 18, 2021.

doi: $10.21037 / \mathrm{tp}-20-353$

View this article at: http://dx.doi.org/10.21037/tp-20-353

^ ORCID: Jia Li, 0000-0002-3606-3949; Qing Lu, 0000-0001-5350-776X; Liangjing Lu, 0000-0001-9116-6038. 


\section{Introduction}

Wilson disease (WD), also known as hepatolenticular degeneration, is a rare autosomal recessive disorder characterized by the impairment of cellular copper excretion. Patients present with hepatic, neurologic and psychiatric manifestations, and presentations vary considerably among patients (1). Due to this variability, making an early diagnosis is difficult, which affects therapeutic outcome and prognosis. Among the signs and symptoms of WD, osteoarticular disturbances, including osteoporosis, osteomalacia, and degenerative changes of joints, have been reported (2). Here, we described a patient with WD who presented with arthralgia and fracture as initial symptoms and carried a novel compound heterozygote of the ATPase copper transporting beta $(A T P 7 B)$ gene. We present the following case in accordance with the CARE reporting checklist (available at http:// dx.doi.org/10.21037/tp-20-353).

\section{Case presentation}

A 13-year-old girl of Chinese Han ethnicity presented to the Department of Rheumatology at Renji Hospital with a 6-month history of joint pain, and limb tremors, clumsy gait, and speech difficulties lasting 3 weeks. A year earlier, the patient had visited a pediatrician for arthralgia in the knees and ankles without triggers. She had no fever, rash, oral ulcer, or low back pain, nor did she report any trauma. Apart from her elder brother, who had undiagnosed muscular atrophy of the limbs, she had no known family history of inherited diseases. The patient underwent a systemic evaluation for differential diagnosis. Physical examination revealed tenderness and swelling in both knees. Laboratory tests showed 1:80 of antinuclear antibodies (ANA, normal value: $<1: 40$ ), with negative anti-double-stranded DNA antibody (antidsDNA), anti-cyclic citrullinated peptide antibody (antiCCP), anti-neutrophil cytoplasmic antibodies (ANCA), human leukocyte antigen B27 (HLA-B27), and rheumatism factors (RF). The erythrocyte sedimentation rate (ESR) and $\mathrm{C}$-reactive protein (CRP) were within normal ranges. Liver and renal functions showed no abnormalities. Plain radiography and magnetic resonance imaging (MRI) of the knees indicated a fracture in the upper end of the tibia as well as osteochondritis of the tibial tubercle (Figure 1). Single-photon emission computed tomography (SPECT) of the knees showed high $99 \mathrm{mTc}$-methylene diphosphonate (99mTc-MDP) uptake in the patient's tibial plateau. A bone marrow biopsy was performed and no abnormalities were found. The patient's cardio-pulmonary function and ophthalmologic examination were normal. She was diagnosed with oligoarticular juvenile idiopathic arthritis (JIA) and received therapy consisting of $10 \mathrm{mg}$ of methotrexate (MTX) every week and diclofenac sodium every day. As the symptoms showed no improvement over 6 months, the treatment was stopped.

Three weeks prior to admission, the patient experienced trembling hands and verbal vagueness. After referral to our department, physical examination showed that she had ataxic dysarthria and essential tremor, which was common with hand and arm involvement. The patient also had increased muscle tone in the left upper limb and a negative pathological reflex. Her laboratory tests revealed a white blood cell count of $4.07 \times 10^{-9} / \mathrm{L}$ (normal value: $4-10 \times 10^{-9} / \mathrm{L}$ ), hemoglobin $122 \mathrm{~g} / \mathrm{L}$ (normal value: $113-151 \mathrm{~g} / \mathrm{L}$ ), platelet count $139 \times 10^{-9} / \mathrm{L}$ (normal value: $101-320 \times 10^{-9} / \mathrm{L}$ ), a negative Coombs direct antibody test, an ESR of $10 \mathrm{~mm} / \mathrm{h}$ (normal value, <20), CRP $1.0 \mathrm{mg} / \mathrm{L}$ (normal value: $<8 \mathrm{mg} / \mathrm{L}$ ), alanine aminotransferase $20 \mathrm{U} / \mathrm{L}$ (normal value: $0-75 \mathrm{U} / \mathrm{L}$ ), aspartate aminotransferase $38 \mathrm{U} / \mathrm{L}$ (normal value: 0-40 U/L), albumin $36.4 \mathrm{~g} / \mathrm{L}$ (normal value: $34-54 \mathrm{~g} / \mathrm{L})$, gamma-glutamyltransferase $56.00 \mathrm{U} / \mathrm{L}$ (normal value: 11-50 U/L), alkaline phosphatase $152 \mathrm{U} / \mathrm{L}$ (normal value: $40-150 \mathrm{U} / \mathrm{L}$ ), direct bilirubin $5.7 \mu \mathrm{mol} / \mathrm{L}$ (normal value: $0.1-5 \mu \mathrm{mol} / \mathrm{L}$ ) and a creatine kinase of $114 \mathrm{U} / \mathrm{L}$ (normal value: 24-248 U/L). Her serum calcium was $2.16 \mathrm{mmol} / \mathrm{L}$ (normal value: $2.08-2.6 \mathrm{mmol} /$ $\mathrm{L}$ ), phosphorus $1.62 \mathrm{mmol} / \mathrm{L}$ (normal value: $0.96-$ $1.62 \mathrm{mmol} / \mathrm{L}$ ), $25-\mathrm{OH}$-vitamin D3 $19.14 \mathrm{ng} / \mathrm{mL}$ (normal value: $>20 \mathrm{ng} / \mathrm{mL}$ ) and parathyroid hormone $25.4 \mathrm{pg} / \mathrm{mL}$ (normal value: $12-88 \mathrm{pg} / \mathrm{mL}$ ). The bone mineral density (BMD) of the patient's hip and lumber spine, as measured by dual-energy $\mathrm{X}$-ray absorptiometry, was normal. However, her abdominal ultrasound revealed liver damage and splenomegaly. No immunological abnormalities were indicated by her laboratory results, except for a positive ANA of 1:80. Pathogen detection tests were negative. MRI of the brain was performed, and $\mathrm{T}_{2}$-weighted MRI and fluid attenuated inversion recovery images (FLAIR) demonstrated hyperintense signals in the brainstem, thalamus, and bilateral basal ganglia (Figure 2), which were suggestive of WD. Ophthalmologic examination with slitlamp examination found Kayser-Fleischer rings. Further laboratory results showed remarkably low ceruloplasmin at $20 \mathrm{mg} / \mathrm{L}$ (normal value: $200-600 \mathrm{mg} / \mathrm{L}$ ). The patient's liver 

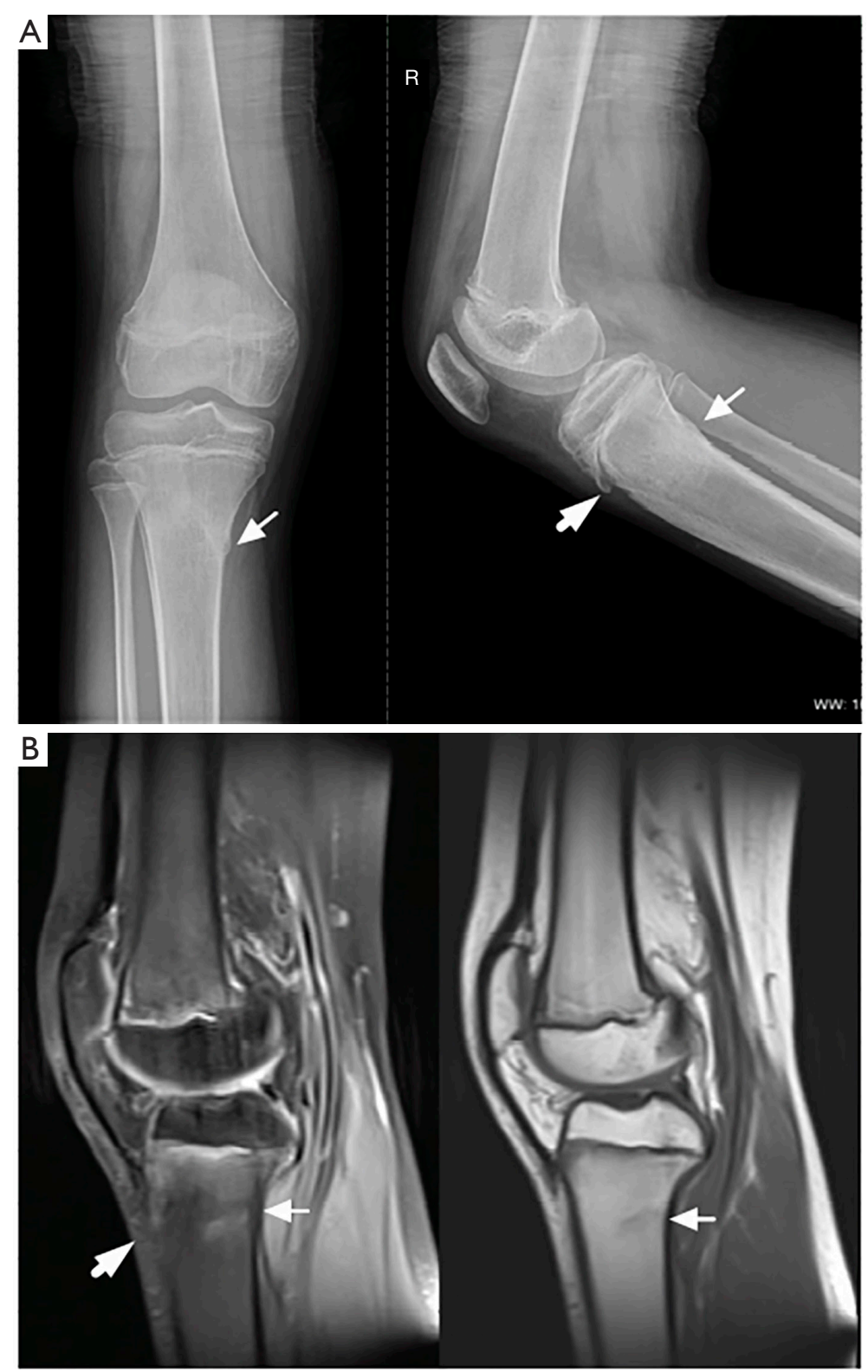

Figure 1 Plain radiographs (A) and magnetic resonance imaging (MRI) (B) images of the right knee indicated a fracture in the upper end of the tibia (small arrows) and osteochondritis of the tibial tubercle (coarse arrows).

biopsy results suggested moderate steatosis of hepatocytes (small vesicular predominance, G1S2) and negative hepatitis $\mathrm{B}$ virus surface antigen (HBsAg), hepatitis B virus e-antigen (HBeAg), and Epstein-Barr virus (EBV). Subsequently, we performed genetic testing for $A T P 7 B$ gene mutations, which are responsible for the pathogenesis of WD (2), using SureSelect Clinical Research Exome v2 (Agilent). A heterozygous mutation of NM_000053.3 (ATP7B): c.3884C $>\mathrm{T}$ p. Ala1295Val was detected and then verified using bi-directional Sanger sequencing. The mutation was found to be inherited from the patient's father (Figure 3). The variant is classified as pathogenic even if it is absent from population databases. The results also suggested the possibility of copy number reduction in exons 10-11 of $A T P 7 B$. Therefore, multiplex ligation-dependent probe amplification (MLPA) was performed, and large fragment heterozygous deletion in exons $10-11$ of $A T P 7 B$ gene inherited from the patient's mother was found (Figure 4). 

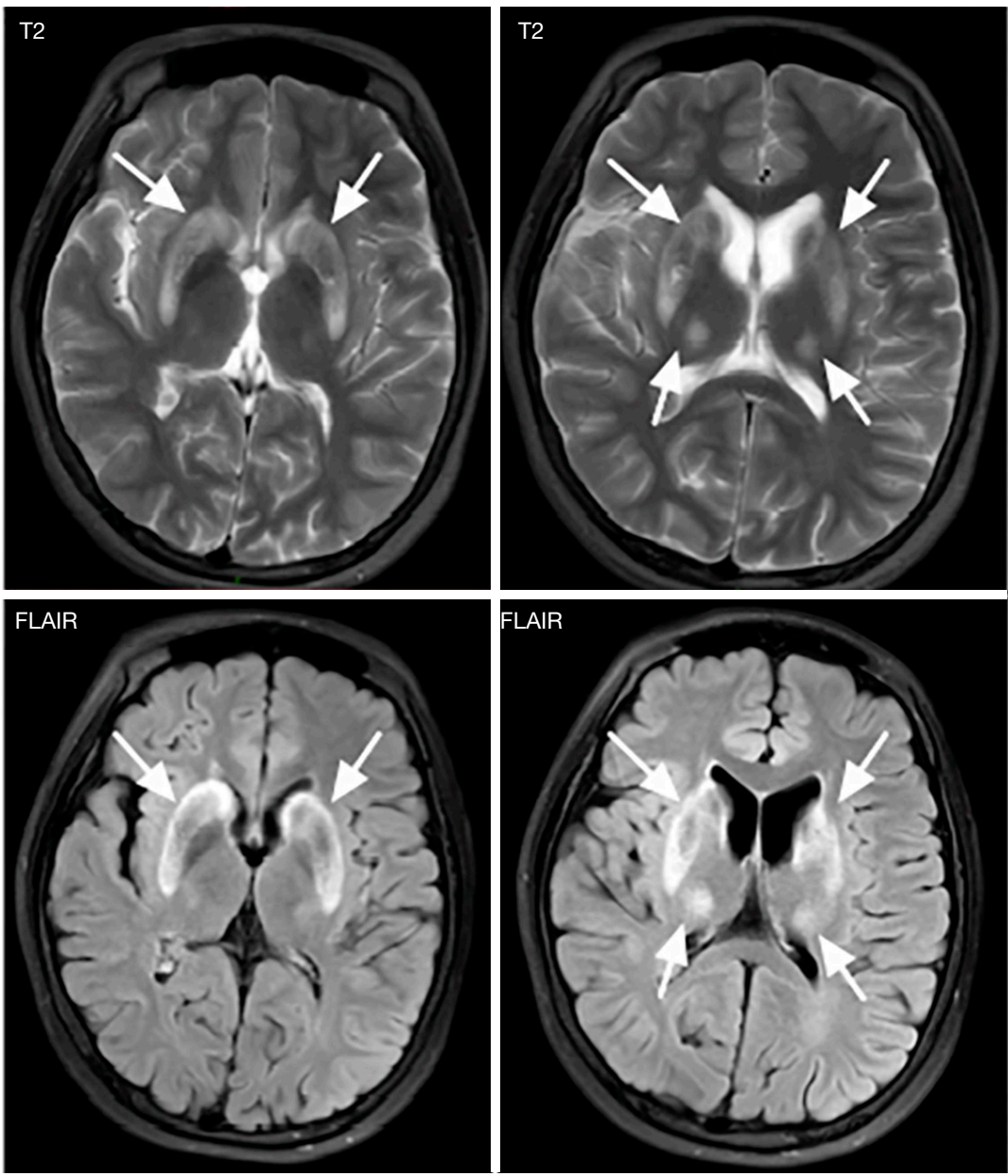

Figure 2 Magnetic resonance imaging (MRI) of the brain demonstrated symmetrical hyperintensity signal on T2-weighted MRI and fluid attenuated inversion recovery images (FLAIR) images in bilateral basal ganglia and thalamus.

A compound heterozygotic mutation leads to defects in ATP7B protein function and copper metabolism. A diagnosis of WD was confirmed.

The patient was treated with oral zinc and Dpenicillamine. At the 1-year follow-up after treatment, her neurologic symptoms and arthralgia had improved. The patient still adheres to this therapy regime currently. Also, the patient's brother underwent whole-exome sequencing, which revealed a compound heterozygous mutation in the Sacsin Molecular Chaperone gene (NM_014363.6:c.8948A>G p.Asn2983Ser and NM_014363.6:c.8948A>G p.Asn2983Ser 014363.5 :c.7466C>A p.Pro2489 His) inherited from their parents. This mutation can lead to spastic ataxia of Charlevoix-Saguenay (SACS, MIM 270550). However, for unknown reasons, he did not pursue further medical testing.
All procedures performed in studies involving human participants were in accordance with the ethical standards of the institutional and/or national research committee(s) and with the Helsinki Declaration (as revised in 2013). Written informed consent was obtained from the patient's parents.

\section{Discussion}

The clinical features of WD are predominantly hepatic, neuropsychic, or a combination of variable symptoms $(3,4)$. The differential diagnosis of WD can be challenging, especially in the early stage of the disease. Musculoskeletal manifestations in WD are often overlooked. Menerey et al. evaluated 24 patients with WD and found minimal radiological findings, including osteophytes, subchondral cysts, chondrocalcinosis, and joint space narrowing (5). 
A

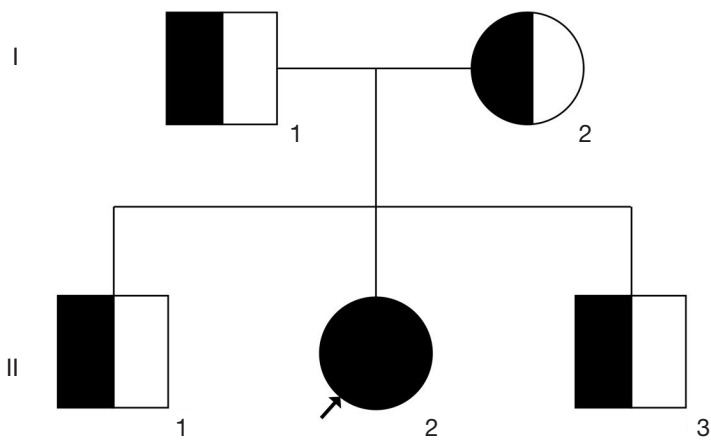

NM_000053.3 (ATP7B): c.3884C>T p. Ala1295Val in exon 18

B

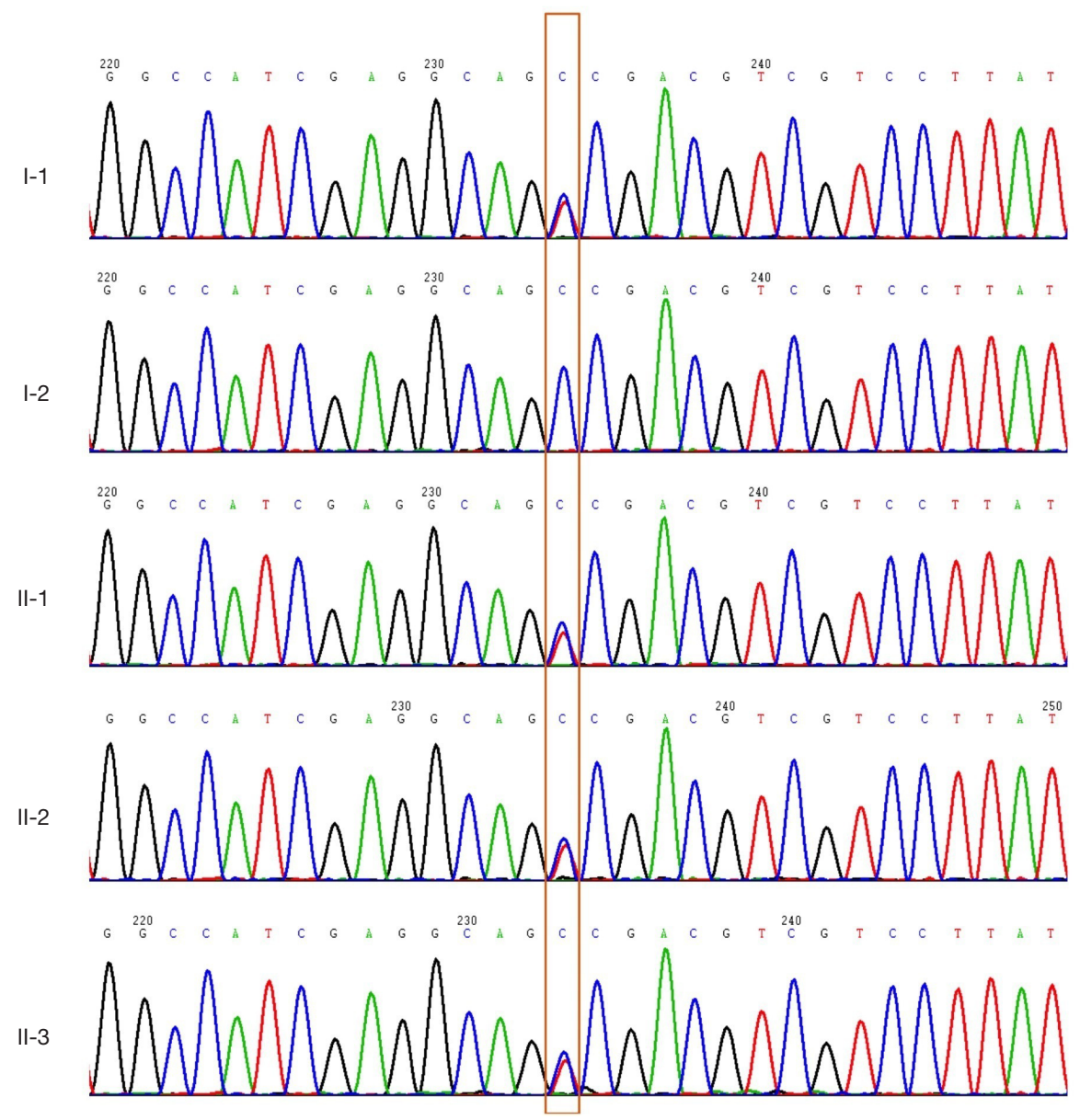

Figure 3 Analysis of NM_000053.3: c.3884C $>$ T mutation in the ATPase copper transporting beta (ATP7B) genes. (A) Family pedigree of the patient; (B) The patient (II-2) carried a heterozygous variant of c.3884C > T verified using Sanger analysis, which was inherited from the patient's father (I-1). 


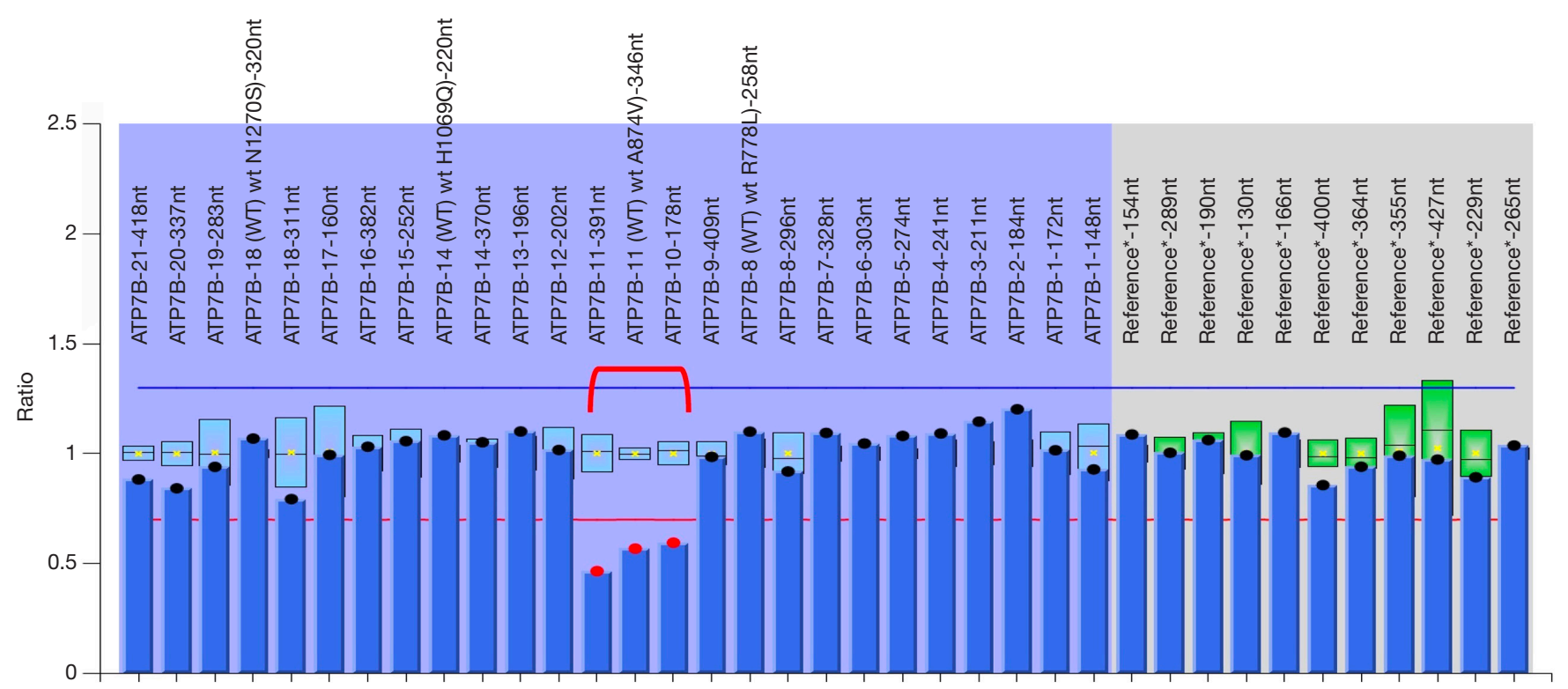

Figure 4 Large fragment heterozygous deletion in exons 10-11 of the $A T P 7 B$ gene using multiplex ligation-dependent probe amplification (MLPA).

Quemeneur et al. summarized musculoskeletal conditions associated with WD. They observed spinal radiological abnormalities, including scoliosis, diffuse bone demineralization, and osteochondritis $(6,7)$. Several groups have reported that decreased BMD and a high prevalence of osteoporosis, especially in the lumbar spine, in patients with WD (8-10). In adults patient with normal BMD, Quemeneur et al. also reported a prevalence of vertebral and peripheral fracture (11).

In our patient, arthralgia, and fracture presented as initial symptoms of WD, which-to the best of our knowledgehas not previously been reported in the literature. The patient did not have osteoporosis, and her serum levels of calcium, phosphorus, and 25-OH-Vitamin D3 were within normal limits. Copper deposition might play a role in the etiology of arthropathy and fracture. However, the mechanisms of osseous abnormality are still unclear. Renal tubular dysfunction and abnormal calcium and phosphorus metabolism have been linked to bone conditions $(2,10)$. Copper is an essential component of proteins and enzymes, and is involved in multiple metabolic pathways. At excess levels, copper induces both the production of reactive oxygen species (ROS) and mitochondrial dysfunction, leading to osteoarticular disorders $(10,12)$.

Furthermore, our patient also had a 1:80 ANA, which more frequently presents in JIA and other autoimmune diseases than in WD. However, our patient did not show other manifestations of rheumatic diseases, and her symptoms were not relieved by MTX.

The Leipzig scoring system, which includes clinical and laboratory testing, is useful in diagnosing WD $(3,13)$. Genetics analysis has the ability to provide early and accurate WD diagnosis. In a clinical series of WD in eastern Spain, Sánchez-Monteagudo et al. identified biallelic $A T P 7 B$ mutations in 30 patients, and in a case, a large deletion of exon 1 with MLPA (14). Our patient harbors a compound heterozygous mutation in the $A T P 7 B$ gene. There are heterozygous mutations of c.3884C>T p.Ala1295 Val in allele 1 and a large heterozygous deletion in exons 10-11 of the $A T P 7 B$ gene in allele 2. To the best of our knowledge, the present study is the first to describe this compound heterozygous mutation. $A T P 7 B$ mutations caused a defect in hepatocellular copper transportation, resulting in the accumulation of copper in various tissues and organs. Xie et al. reported that skeletal copper content increased 4 times in patients with WD, which might lead to bone and joint changes (15).

It is also interesting that the patient's brother demonstrated additional mutations in the SACS gene, which might cause SACS. It is uncommon for siblings to have different genetic diseases in one family.

Our case suggests that, due to their rarity, 
musculoskeletal presentations of WD are usually neglected in WD patients at an early stage. WD should be considered in adolescents with unexplained arthralgia of the large joints. Patients with WD may demonstrate abnormal autoimmune features, such as a low titer of positive ANA or arthritis in large joints. Early diagnosis and therapy can aid in improving the prognosis of patients with WD.

\section{Acknowledgments}

We thank Drs. Ru-en Yao and Pengjie Wan from Shanghai Children's Medical Center, Shanghai Jiao Tong University School of Medicine, for performing Sanger sequencing. We thank R. Scott and J. Reynolds, from AME editing service (http://editing.amegroups.com), for English language editing of the manuscript.

Funding: This study was supported by the National Key Research and Development Program of China (2017YFC0909002) and the National Natural Science Foundation of China (81102267).

\section{Footnote}

Reporting Checklist: The authors have completed the CARE reporting checklist. Available at http://dx.doi.org/10.21037/ tp-20-353

Peer Review File: Available at http://dx.doi.org/10.21037/tp20-353

Conflicts of Interest: All authors have completed the ICMJE uniform disclosure form (available at http://dx.doi. org/10.21037/tp-20-353). The authors have no conflicts of interest to declare.

Ethical Statement: The authors are accountable for all aspects of the work in ensuring that questions related to the accuracy or integrity of any part of the work are appropriately investigated and resolved. All procedures performed in studies involving human participants were in accordance with the ethical standards of the institutional and/or national research committee(s) and with the Helsinki Declaration (as revised in 2013). Written informed consent was obtained from the patient's parent.

Open Access Statement: This is an Open Access article distributed in accordance with the Creative Commons Attribution-NonCommercial-NoDerivs 4.0 International
License (CC BY-NC-ND 4.0), which permits the noncommercial replication and distribution of the article with the strict proviso that no changes or edits are made and the original work is properly cited (including links to both the formal publication through the relevant DOI and the license). See: https://creativecommons.org/licenses/by-nc-nd/4.0/.

\section{References}

1. Członkowska A, Litwin T, Dusek P, et al. Wilson disease. Nat Rev Dis Primers 2018;4:21.

2. Dzieżyc-Jaworska K, Litwin T, Członkowska A. Clinical manifestations of Wilson disease in organs other than the liver and brain. Ann Transl Med 2019;7:S62.

3. Liver EAfSo. EASL Clinical Practice Guidelines: Wilson's disease. J Hepatol 2012;56:671-85.

4. Bandmann O, Weiss KH, Kaler SG. Wilson's disease and other neurological copper disorders. Lancet Neurol 2015;14:103-13.

5. Menerey KA, Eider W, Brewer GJ, et al. The arthropathy of Wilson's disease: clinical and pathologic features. J Rheumatol 1988;15:331-7.

6. Quemeneur AS, Trocello JM, Ea HK, et al. Miscellaneous non-inflammatory musculoskeletal conditions.

Musculoskeletal conditions associated with Wilson's disease. Best Pract Res Clin Rheumatol 2011;25:627-36.

7. Rosen JM, Kuntz N, Melin-Aldana H, et al. Spasmodic muscle cramps and weakness as presenting symptoms in Wilson disease. Pediatrics 2013;132:e1039-42.

8. Selimoglu MA, Ertekin V, Doneray H, et al. Bone mineral density of children with Wilson disease: efficacy of penicillamine and zinc therapy. J Clin Gastroenterol 2008;42:194-8.

9. Hegedus D, Ferencz V, Lakatos PL, et al. Decreased bone density, elevated serum osteoprotegerin, and beta-crosslaps in Wilson disease. J Bone Miner Res 2002;17:1961-7.

10. Weiss KH, Van de Moortele M, Gotthardt DN, et al. Bone demineralisation in a large cohort of Wilson disease patients. J Inherit Metab Dis 2015;38:949-56.

11. Quemeneur AS, Trocello JM, Ea HK, et al. Bone status and fractures in 85 adults with Wilson's disease. Osteoporos Int 2014;25:2573-80.

12. Li S, Wang M, Chen X, et al. Inhibition of osteogenic differentiation of mesenchymal stem cells by copper supplementation. Cell Prolif 2014;47:81-90.

13. Xuan A, Bookman I, Cox DW, et al. Three atypical cases of Wilson disease: assessment of the Leipzig scoring system in making a diagnosis. J Hepatol 2007;47:428-33. 
14. Sánchez-Monteagudo A, Álvarez-Sauco M, Sastre I, et al. Genetics of Wilson disease and Wilson-like phenotype in a clinical series from eastern Spain. Clin

Cite this article as: $\mathrm{Li} \mathrm{J,} \mathrm{Lu} \mathrm{Q,} \mathrm{Yu} \mathrm{J,} \mathrm{Ji} \mathrm{M,} \mathrm{Lu} \mathrm{L.} \mathrm{Osteoarticular}$ manifestations as initial symptoms of WD with novel compound heterozygote mutations in the $A T P 7 B$ gene: a case report. Transl Pediatr 2021;10(4):1026-1033. doi: 10.21037/tp-20-353
Genet 2020;97:758-63.

15. Xie YZ, Zhang XZ, Xu XH, et al. Radiologic study of 42 cases of Wilson disease. Skeletal Radiol 1985;13:114-9. 